DOI: $10.1515 / \mathrm{rrlm}-2017-0019$

\title{
Involvement of inflammatory markers in pathogenesis of venous thromboembolism
}

\author{
Alexandra Florina Cocoi ${ }^{*}$, Dana Pop ${ }^{2}$, Mihai Cocoi ${ }^{3}$, \\ Adela Mihaela Serban ${ }^{3}$, Luminita Animarie Vida-Simiti ${ }^{1}$ \\ 1. Spitalul Clinic Judetean de Urgenta Cluj-Napoca, UMF "Iuliu Hatieganu" Cluj-Napoca, Romania \\ 2. Spitalul Clinic de Recuperare Cluj-Napoca, UMF "Iuliu Hatieganu" Cluj-Napoca, Romania \\ 3. Institutul Inimii Cluj-Napoca, UMF "Iuliu Hatieganu" Cluj-Napoca, Romania
}

\begin{abstract}
Inflammation of the venous wall is involved in thrombogenesis, thrombus resolution, wall remodeling and the post-thrombotic syndrome. Different mechanisms are involved in both arterial and venous thrombosis and patients with atherothrombosis hold a higher risk of venous thrombosis. Although inflammation may represent the connection between arterial and venous thrombosis, it is not yet sure if it is the cause or consequence of venous thrombosis. Consequently, the relationships between inflammation markers as indicators of the inflammatory process and clinical venous thromboembolism need to be investigatd. For example, inflammation mediators such as the pro-inflammatory cytokines interleukin 8 (IL-8), IL-6, monocyte chemotactic protein 1 (MCP-1), C Reactive Protein (CRP), vascular cell adhesion molecule 1 (VCAM-1), intercellular adhesion molecule 1 (ICAM-1), matrix metalloproteinases and tumor necrosis factor alpha (TNF alpha) are all involved in thrombogenesis. Studies of venous thromboembolism on animal models proved that there are specific phases of the inflammatory process in venous thromboembolism and thrombus resolution. Knowing the molecular and immunologic mechanisms, identifying and understanding the inflammation markers which are relevant for venous thrombosis, can help to target specific pathways and to develop future therapies of this disease.
\end{abstract}

Keywords: thrombembolism, markers, inflammation, thrombus, venous thrombosis

Received: $21^{\text {st }}$ February 2017; Accepted: 28 ${ }^{\text {th }}$ May 2017; Published: $16^{\text {th }}$ July 2017

\section{Introduction}

Inflammation was recently established as a possible mechanism that determines venous thrombosis formation. Recent data indicates that thrombus formation inside a vein is determined by inflammation of the vascular wall and that the systems of coagulation and inflammation are involved at different levels in the same activation pathway. The first step in the formation of thrombus is activation of platelets, leukocytes, and endothelial cells, followed by initiation of inflammation and generation of microparticles, induction of tissue factor, triggering the coagu-

\footnotetext{
*Corresponding author: Alexandra Florina Cocoi, Spitalul Clinic Judetean de Urgenta Cluj-Napoca Cluj-Napoca, Romania, e-mail: alexandraflorina.popa@gmail.com
} 
lation system. Inflammation is involved not only in thrombogenesis, but also in thrombus resolution and remodeling of the venous wall. However, the real connection between markers of inflammation and venous thromboembolism is not completely understood. By knowing the molecular and immune mechanisms and identifying the inflammation markers relevant for venous thrombosis we might identify targets for future therapies of this disease [1].

\section{Inflammation - the connection between arterial and venous thrombosis}

Deep venous thrombosis is a multifactorial condition. The physiopathological mechanisms of venous thromboembolism include endothelial destruction, stasis and hypercoagulability [1]. In the last years, inflammation has been acknowledged as a possible mechanism through which several risk factors determine the formation of venous thrombus. Animal studies demonstrated that thrombosis leads to an inflammatory response of the vein wall, which implies activation of neutrophils and expression of selectins, inflammatory cytokines and cell adhesion molecules [2]. It was proven that leukocytosis contributes to venous thrombosis by destruction of endothelium, and leukocytosis together with the systemic inflammatory response are important factors in determining the short term prognosis of venous thromboembolism $[3,4]$.

An important argument in favor of the impact of inflammation in venous thromboembolism is the elevated risk of venous thrombosis and embolism in different inflammatory diseases, such as vasculitis, sarcoidosis, rheumatoid arthritis, inflammatory myopathies, gout, celiac and inflammatory bowel disease and perhaps other autoimmune diseases [5-12].

Clasically, the physiopathology of thrombosis was separated into the arterial and venous thrombosis. Venous thrombosis was associated with formation of red thrombi, rich in fibrin and erythrocytes, while white arterial thrombi are rich in platelets. Venous thrombi contain two regions: one including mainly erythrocytes and fibrin (red thrombus), while the other is predominantly made of aggregated platelets (white thrombus). Components rich in fibrin have a role in attaching thrombi to the arterial wall, while the components rich in platelets have been detected far from the attachment site. These observations suggest that coagulation precedes platelet activation and aggregation, fibrin formation being the first event occurring in venous thrombosis [1].

In the last decades, the concept of different mechanisms involved in both arterial and venous thrombosis (a fundamental role of inflammation) was partially demonstrated by the evidence suggesting that patients with atherothrombosis hold a higher risk of venous thrombosis. Atherosclerosis is associated both with activation of platelets and of the coagulation cascade, which can lead to thrombotic complications. Presently, it is extensively recognized that, initiation of the coagulation cascade with generation of thrombin and deposit of fibrin as a consequence of inflammation is perhaps implicated in both arterial and venous thrombosis pathogenesis $[1,13]$.

Furthermore, the fact that inflammation is a key process is suggested by the relationship between arterial and venous thrombosis and several common risk factors: old age, high BMI (body mass index), hypercholesterolemia, arterial hypertension, smoking, diabetes mellitus, lupus anticoagulant and hyperhomocysteinemia. Moreover, the metabolic syndrome - abdominal obesity, insulin resistance, arterial hypertension, hypertriglyceridemia, low HDL (high density lipoprotein) cholesterol - an important risk factor of the atherosclerotic disease, has been recently associated with venous thromboembolism [4-15].

It has also been demonstrated that there is a relationship between subclinical and clinical 
atherosclerotic disease and venous thromboembolism. Patients with primary venous thrombotic events have significantly higher prevalence of carotid plaques, carotid intima-media thickness and number of carotid segments affected compared with those with secondary events of same sex and age and case control patients with no thrombosis. In patients with idiopathic venous thrombosis, endothelial dysfunction has also been noticed $[1,15]$.

It has been proven that there is a link between manifest atherosclerotic disease and venous thromboembolism. The survivors of a myocardial infarction had a 2.1 higher risk of venous thromboembolism in the follow up period, but the risk was 5 times higher in the first 90 days after the event. Furthermore, an association has been found between venous thromboembolism and peripheral artery disease of the inferior limbs. The link between arterial and venous disease has been confirmed by a study that proved that patients with venous thromboembolism hold a risk of arterial events almost 3 times higher compared to the control group [1,13].

Drugs used in the prevention of atherosclerotic cardiovascular events are also useful in the prevention of venous thrombosis. A 50\% reduction of venous thromboembolism risk has been associated with the use of statins $[1,15,16]$. The LDL (low density lipoprotein) cholesterol levels did not influence thromboembolic risk, therefore the protective effect of statins may be due to their pleiotropic, antithrombotic and anti-inflammatory properties [15] and improvement in blood rheological properties [1]. It has also been demonstrated that the risk of venous thromboembolism in patients with clinical coronary heart disease is lowered by long term aspirin treatment $[1,17]$.

Both arterial and venous thromboembolism associate abnormal concentrations of vascular injury proteins, vascular stress markers, acute inflammation markers, myocardial necrosis markers, mediators of angiogenesis and apoptosis. Biological markers implicated in these processes can improve diagnostic accuracy and determination of disease prognosis in emergency situations [18].

\section{Interaction between inflammation and thrombosis}

Venous thrombosis is increasingly considered an inflammatory disease, this being proven by multiple studies which suggested a link between inflammatory markers and venous thromboembolism. During inflammation, the hemostatic equilibrium can be influenced, determining the increase in procoagulant and reduction of anticoagulant factors. Fibrinolytic activity is also inhibited by inflammatory mechanisms, and the mediators of inflammation seem to increase platelet activity. Inflammation determines activation of coagulation, and coagulation affects considerably the inflammatory activity, due to the fact that thrombin, which is a procoagulant, can stimulate multiple inflammatory pathways. Thus, the formation of thrombus promotes the inflammatory response, which subsequently, stimulates the pro-thrombotic factors. These affirmations are supported by the fact that thrombosis can be prevented by natural anticoagulants, such as protein $\mathrm{C}$ and $\mathrm{S}$, antithrombin III, which can also reduce inflammatory activity [1,2,15,19-21].

As cytokines' levels were measured in all studies after the onset of thrombosis, it can be presumed that the inflammatory reaction was a consequence, rather than a cause of the thrombotic events. However, if considering inflammation as a consequence of thromboembolism, there should be a correlation between the time passed since the thrombotic event and the decrease of the cytokine level, which was not demonstrated. An increase in the inflammatory markers, which might reflect an independent inflammatory pro- 
cess or a subclinical persistent inflammation, may be secondary to other diseases associated with venous thrombosis, such as post-thrombotic syndrome or occult cancer. [15,17,19-21].

Chronic venous insufficiency consists of detrimental changes of vein wall and destruction of venous valves after venous thrombosis, all being the result of the interaction between thrombosis and inflammation (phlebitis). Even though the relation between inflammation and post-thrombotic syndrome is not yet fully understood, the results of a study suggest that inflammation, which includes both the vein wall and the perivascular area, can play a role in the incomplete resolution of thrombus. The two processes involved in the development of post-thrombotic syndrome are the persistent obstruction (subsequent to incomplete thrombus lysis and fibrosis of the vein wall as a response to thrombus and vein wall inflammation), and blood reflux (as a result of valve incompetence). Both mechanisms determine venous hypertension and post-thrombotic syndrome [21].

The control of inflammation limits the destruction of the vein wall determining a reduction in the clinical manifestations of chronic venous insufficiency and the recurrence of thrombosis. Patients with deep venous thrombosis can benefit from treatment with anti-inflammatory agents or low molecular weight heparins, due to the fact that they hold both anti-inflammatory and anticoagulant properties [19-21].

\section{The main inflammatory markers}

The main mediators of inflammation that induce the activation of coagulation are the pro-inflammatory markers belonging to a small family of chemotactic cytokines implicated in leucocyte trafficking, such as IL (interleukin)-6, IL-8, MCP (monocyte chemoattractant protein)-1, which generate expression of tissue factor in monocytes, key inductors of blood coagulation $[1,15]$.
The pro-coagulant factors, fibrinogen and factor VIII are acute phase proteins whose synthesis is amplified by IL-6. IL-8 is a chemoattractant and a neutrophil activator, and the chemotaxis of the monocytes to the endothelium is mediated by $\mathrm{MCP}-1$, both contributing to a pro-coagulant status by activating the endothelium $[1,15,17,21]$.

The cytokine neutralizing antibodies and adhesion molecule antibodies were efficient in limiting early neutrophil and late monocyte and macrophage response. [19-21]

\section{Il-8 and IL-6}

It was demonstrated that patients with high serum levels of these inflammatory cytokines present a risk 2-3 times higher to develop venous thrombosis [1,17,19-22]. Moreover, plasma concentrations of these immune mediators were increased in patients with recurrent venous thrombosis. For IL-8 in particular, the risk seems to arise in parallel with an increase in the serum levels [15]. Only IL-6 levels demonstrated a significant relation to the extension of thrombus $[18,23]$. Furthermore, levels of IL-6 and IL- 8 are associated with high risk of a first venous thromboembolism [15,23].

Compared to healthy subjects, patients with deep venous thrombosis present a systemic inflammatory response with high plasma levels of IL- 6 and IL- 8 in the acute phase of the disease, maximal levels being reached in day 2 after the induction thrombosis $[2,18,23]$. During follow up, levels of IL-8 remained high, but IL-6 dropped in evolution $[1,17,18,23]$.

Multiple studies showed high levels of IL-8 in association with both acute and chronic inflammatory conditions. Pro-inflammatory cytokines such as IL-1beta and TNF (tumor necrosis factor) alpha, bacterial and viral agents can stimulate the production of IL-8. It is produced by many types of cells, including neutrophils, monocytes, endothelial cells, epithelial cells and fibroblasts. A number of mechanisms can explain 
the connection between IL- 8 concentrations and venous thrombosis. Firstly, it has been proven that IL-8 determines the expression of tissue factor in monocytes, this being an important inductor of blood coagulation. Secondly, IL-8 induces leukocyte recruitment, the first cells that adhere to the vein endothelium in deep venous thrombosis. Moreover, IL-8 might contribute to the generation of a procoagulant surface through monocyte adhesion to the vascular endothelium [22].

\section{CRP}

High levels of $\mathrm{C}$ reactive protein (CRP) in venous thrombosis, secondary to acute phase reactions [16], induce the expression of tissue factor in monocytes, smooth muscle cells and endothelial cells $[13,24]$. CRP levels have been directly proportional to the thrombotic load, the extension and volume of thrombus [25]. While some studies did not show a link between CRP levels and a future venous thrombotic event, others demonstrated a significant correlation between high levels of CRP and further development of thromboembolism [1,2,15,24]. Although previous studies showed that at the moment of diagnosis there are high levels of CRP, it was proven that plasma CRP levels have no utility in infirming or confirming this diagnosis in patients with clinical suspicion of deep vein thrombosis $[1,2,18,25]$. Nevertheless, CRP plays a role in assessing the mortality risk and early complications [24,25].

\section{TNF alpha}

According to some studies, TNF alpha is involved in the thrombotic risk, as patients with increased levels of TNF alpha had a two times higher risk for developing venous thrombotic events [1,17]. Meanwhile, other studies have shown a non-significant difference in TNF alpha concentrations between the groups of patients with and without this pathology [2]. TNF seems to play a primordial role not only in the mediation of early inflammatory response and in the extravasation of neutrophils, but also in the late inflammatory response. TNF is also an important mediator that determines ICAM (intercellular adhesion molecule)-1 expression on the surface of endothelial cells and facilitates neutrophil, monocyte and lymphocyte adhesion at the inflammation site [24,25].

\section{IL-10}

While some studies showed no association [26] or a weak association [17] between venous thromboembolism and IL-1beta, the genetic variety of IL-1beta and IL-10 can influence the risk of idiopathic venous thromboembolism. Activated macrophages produce IL-1beta as an important mediator of the inflammatory process, but also a procoagulant factor, involved in cell proliferation, differentiation, apoptosis and expression of tissue factor in endothelial and monocytic cells [24,25]. IL-10 levels were high in the vein wall at the time of venous thrombosis, being produced especially by monocytes and, at a lesser extent, by lymphocytes. By neutralizing IL-10, the inflammatory process increased, while a supplementation of recombinant IL-10 showed a reduction in inflammation $[24,25]$. These data prove the anti-inflammatory properties of IL-10 and suggest a protective effect against thrombus formation [1,17,24-26]. Therefore, IL-10 can be used as a therapeutic agent in the treatment of deep venous thrombosis [26]. The IL-10 G 10 allele has been more frequently found in recurrent venous thromboembolism, suggesting that it can be considered as an independent risk factor for the prediction of recurrence $[24,25]$.

\section{VCAM-1,ICAM -1, VEGF}

Some studies did not account for an association between venous disease and VCAM (vascular cell adhesion molecule)-1, [2,26] ICAM-1 or VEGF (vascular endothelial growth factor) 
[26]. However, one study showed that the levels of VCAM-1 were increased in patients with deep vein thrombosis, suggesting a possible role in the pathogenesis of this condition [24,25]. On day 6 after thrombus formation, the intervention of ICAM -1 was proven by the fact that the most efficient antibody that can attenuate leukocyte extravasation was anti-ICAM 1 , reducing the number of neutrophils and monocytes at the thrombotic site [26].

\section{The phases of the inflammatory process in venous thromboembolism}

Studies of venous thromboembolism on animal models proved an association between thrombosis and parietal inflammation (both of the vein wall and pulmonary arteries) [18].

\section{The pulmonary artery wall}

At the onset, there are almost no inflammatory cells in the walls of pulmonary arteries. In the next 3 hours after a pulmonary embolism, proof of cell infiltration is found at the external border of the pulmonary artery wall. The leukocyte influx, both in the pulmonary arteries and in the pulmonary parenchyma, occurs after the pulmonary embolism (at approximately one day). There is a 38 times increase in the leukocyte number compared to control animals, most of them being neutrophils, followed by macrophages. By day 1 or 2 after a pulmonary embolism, a higher infiltration rate with neutrophils and macrophages is noted in the pulmonary artery wall; moreover, there are granulocytes in the vasa vasorum. Two days after the pulmonary embolism, the total leukocyte count is 329 higher than normal, neutrophils being the most numerous. After 4 days, most of the thrombi are lysed and a considerable reduction of inflammatory cell infiltration is noted. The total leukocyte count reduces at 45 times the normal value. At 14 days after the pulmonary embolism, the wall of the pulmonary arteries is similar to the onset of pulmonary embolism, with no significant differences concerning the leukocyte number $[27,28]$. Beginning with the fourth day after the pulmonary embolism, an increase in the intima thickness is found, associated with an increase in the cellularity within the intimal layer compared to control animals. A significant growth of intima/media ratio is described at 4, 6 and 14 days after the pulmonary embolism. Intimal hyper-cellularity persisted up to 14 days after the acute episode, most likely due to smooth muscle cells [28].

\section{The venous wall}

Regarding the venous wall, there are few inflammatory cells at the onset. One hour after the thrombosis, neutrophils start to align at the interface between thrombus and venous wall or in the vasa vasorum, but very few in the interior of the wall. At the end of the first day, multiple neutrophils are found in the vein wall, penetrating both from the luminal and adventitial side of the wall. The trans-endothelial migration leads to cell agglomeration in certain parts and a removal of endothelial cells and exposure of the basal membrane in other parts, thus creating a thrombogenic surface. The extravasation of neutrophils and afterwards of monocytes, macrophages and lymphocytes out of the venous wall is established by a chemotactic gradient. On day 3 , while the thrombus matures, the monocytes and lymphocytes join the neutrophils, together with fibroblastic cells that participate in organizing the thrombus and vein wall. In animal models, the macrophages are the most numerous leukocytes at 3 days after thrombus formation. On day 6 , the thrombus undergoes organization, the interface between thrombus and vein wall being less and less defined [24,25,27,28].

\section{The pulmonary parenchyma}

In the pulmonary parenchyma, there are macrophages and few neutrophils at onset. After 
the pulmonary embolism, there is a rise in the number of macrophages compared to controls. This modification is first noted at 3 hours and persists up to 4 days after the pulmonary embolism. In contrast to the arterial pulmonary wall, neutrophils are present in the distal segments of pulmonary vessels in the counter-lateral lung and with a significant increase in the total number of macrophages and leukocytes at 2 and 4 days after the pulmonary embolism [27-29].

\section{The inflammatory markers}

Regarding the levels of the cytokines and chemokines in the pulmonary artery wall, only the MCP-1 concentration is increased 2 days after the pulmonary embolism, thus preceding the macrophages influx. MCP-1 levels are also significantly higher compared to the baseline value at day 1 after the pulmonary embolism in the counter-lateral pulmonary artery [28]. These changes differ from the response to a thrombus development in a vein. In this case, TNF-alpha, IL-6, MCP-1, cell adhesion molecules and P-selectin levels increase. Importantly, P-selectin is not found in the pulmonary artery wall after venous thromboembolism, a different aspect from the genesis of deep venous thrombosis, where $\mathrm{P}$-selectin is essential in mediating the interaction between leukocytes, endothelial cells and platelets [28].

Right ventricle dysfunction, an important risk factor for unfavorable clinical evolution after a pulmonary embolism, is mediated by cardiac inflammation dependent on neutrophils. Histological analysis of the right ventricular muscle in subjects with fatal pulmonary thromboembolism show a massive accumulation of neutrophils and monocytes/macrophages, but no histological proof of infarction. Selective anti-inflammatory therapies targeted against neutrophil chemo-attractants lead to a reduction in cardiac inflammation and in right ventricular dysfunction in pulmonary embolism [22].

\section{The role of inflammatory markers in thrombus resolution}

The resolution of pulmonary thrombus is produced by endogenous thrombolysis, fragmentation, organization or re-canalization. The organization of thrombus implies neo-vascularization, which can be induced by the chemokines and growth factors that are present at the time of thrombotic resolution. Thrombus re-canalization was correlated with the formation of intimal fibrosis and intraluminal fibrous bands and can lead to pulmonary hypertension associated with pulmonary embolism [1,30,31].

While the thrombus ages, in the first 24-72 hours, there is a transition towards a cellular phase of the inflammation, and the balance inclines towards the chemokines involved in the chemotaxis of neutrophils and monocytes. The cellularity of the thrombus is modified by IL8 , resulting in day 4 in an increased number of neutrophils, monocytes, fibroblasts, endothelial cells, especially in the area close to the neo-vessels [30,31]. IL-8 represents a potent angiogenic factor and amplifies the organization of the thrombus. The recruitment of granulocytes and angiogenesis are both mediated by IL- 8 . The specific contribution of neutrophil influx in angiogenesis is still not defined, but the depletion of granulocytes is associated with larger thrombi with a higher concentration of collagen, while the supplementation with IL-8 is associated with smaller thrombi, concomitant with an increase in neo-vascularization [32]. Published data suggest the possibility of using angiogenic chemokines, such as IL-8, for the treatment of venous thrombosis because of its favorable effect on thrombus stabilization and neo-vascularization, thus promoting the early recanalization and restoring the perfusion, especially when injected directly into the thrombus [32,33].

The macrophage and monocyte influx is essential for angiogenesis, having an import- 
ant contribution to the cellular activity of the thrombus. The macrophages recruited at the site of embolism and the monocytes which differentiate into tissue macrophages secrete an enhanced amount of pro-fibrinolytic proteins, which contribute to the resolution of pulmonary embolism and deep vein thrombosis. Monocyte influx in the thrombus reaches a peak on day 8 after thrombogenesis and correlates with high levels of MCP-1. The monocytes can conduct neovascularization by synthesizing factors which upregulate the cell adhesion molecules, crucial to the formation of new vessels: tissue factor plasminogen activator, urokinase and urokinase receptors. Macrophage function can be shifted by MCP-1 towards fibrinolysis. Thus, the monocyte influx and MCP-1 in days 8 and 12 are associated with an increase in thrombus resolution [1,28-32]. The intimal layer of the pulmonary arteries thickens and becomes hypercellular immediately after an increase of the MCP-1 levels and macrophage population and treatment with anti-MCP-1 antibodies inhibits intimal hyperplasia [28].

Simultaneously with the early biomechanical injury in deep venous thrombosis, an increase of profibrotic mediators is noticed in the vein wall and thrombus, this including the transforming growth factor (TGF) beta, IL-13 and MCP-1. IL13 is secreted by activated T-helper lymphocytes and promotes the expression of MCP-1. It is interesting to note that high concentrations of IL13 and MCP-1 were found in pulmonary tissue after a pulmonary embolism and were associated with significant intimal hyperplasia. Although MCP-1 may increase fibrosis, it may also accelerate the resolution of thrombus when it is present at this level [16,30]. TGF beta is being activated by natural thrombolysis and is also present in the thrombus, promoting fibrosis of the vein wall. Therefore, early injury of the vein wall is associated with an active remodeling of the matrix which seems to promote fibrosis [30,34].
The resolution of thrombus implies both pro-fibrotic factors and collagen deposits, along with the expression and activation of matrix metalloproteinases (MMPs) [1]. The role of leukocytes in natural thrombus resolution is demonstrated by the fact that they invade the thrombus in a unique sequence. Neutrophils are essential for the early resolution of thrombus by promoting fibrinolysis and collagenolysis. In an animal model, neutropenia was associated with larger thrombi in the second and seventh day and was correlated with higher degrees of fibrosis in the thrombus and significantly lower serum levels of plasminogen activators such as urokinase and MMP-9. Early collagenolysis seems to take place in the first 7 days, as suggested by a high rigidity in the vein wall and persists up to 14 days, being accompanied by high activity of MMP-2 and MMP-9 [35].

While the matrix metalloproteinases have been recognized as key components in physiological or pathological breakout of the extracellular matrix and also in pulmonary vascular remodeling in thromboembolic pulmonary hypertension, studies have shown that they can also modulate vascular reactivity. Vascular MMP-2 and neutrophil MMP-9 seem to promote vasoconstriction and breakdown of vasodilatation substances. The high expression and activity of these matrix metalloproteinases is found during inflammation and pulmonary aggression. Knowing all this, high activity of MMP-2 and MMP-9 reflect the recruitment and the migration of inflammatory cells into the pulmonary tissue. Therefore, the most probable mechanism behind this high MMP-9 activity after an acute pulmonary embolism implies an early neutrophil influx into the pulmonary artery and fast release of some granules which contain high quantities of MMP-9. The activated neutrophil generates reactive oxygen species, which subsequently release MMPs. Consequently, an increase in the oxidative stress 
in pulmonary embolism activates MMP-2 and MMP-9. [36].

Concerning the relation between inflammation and fibrosis, current studies suggest that the inhibition of the inflammatory response can diminish the fibrosis of the vein wall. In a comparative study between low weight heparins and oral $\mathrm{P}$ selectin inhibitors administered 2 days after the thrombosis, it was revealed that $\mathrm{P}$ selectin inhibitors significantly reduce the injury of the vein wall, independently of the size of thrombus [34,35].

\section{Conclusions}

The inflammatory response can determine hypercoagulability, increasing the chance of venous thrombosis, but the complex relationship between venous thromboembolism and inflammation is not yet fully understood. Future studies may demonstrate the probable predictive value of inflammatory markers for diagnosis and evolution of this disease (severity, risk of recurrence). Moreover, if inflammation proves to be a cause of venous thrombosis, identifying high levels of inflammatory markers that are relevant for venous thromboembolism would offer new therapeutic targets based on drugs with anti-inflammatory properties.

\section{Conflict of interest statement}

We have no conflict of interest to declare.

\section{References}

1. Poredos P, Jezovnik MK. The Role of Inflammation in Venous Thromboembolism and the Link Between Arterial and Venous Thrombosis. Int Angiol 2007;26:30611.

2. Fox EA, Kahn SR. The relationship between inflammation and venous thrombosis. A systematic review of clinical studies. Thromb Haemost 2005;94:362-5. DOI: 10.1160/th05-04-0266

3. Jo JY, Lee MY, Lee JW, Rho BH, Choi WI. Leukocytes and systemic inflammatory response syndrome as prognostic factors in pulmonary embolism patients. BMC Pulm Med [Internet] 2013 [cited 2017 Jan 02];13:74[8p].

4. Zhang Y, Yang Y, Chen W, Liang L, Zhai Z, Guo L, et al. Hypertension associated with venous thromboembolism in patients with newly diagnosed lung cancer. Sci Rep [Internet] 2016 [cited 2017 Jan 02];6:19603[7p]. Available from: PubMed Central.

5. Unizony S, Lu N, Tomasson G, Zhang Y, Merkel PA, Stone JH, et al. Temporal Trends of Venous Thromboembolism Risk Before and After Diagnosis of Giant Cell Arteritis. Arthritis Rheumatol 2017 Jan;69(1):17684. DOI: $10.1002 / \operatorname{art} .39847$

6. Goljan-Geremek A, Geremek M, Puscinska E, Sliwinski P. Venous thromboembolism and sarcoidosis: co-incidence or coexistence? Cent Eur J Immunol 2015;40(4):477-80. DOI: 10.5114/ceji.2015.56972

7. Lee DK, Kim HJ, Lee DH. Incidence of Deep Vein Thrombosis and Venous Thromboembolism following TKA in Rheumatoid Arthritis versus Osteoarthritis: A Meta-Analysis. PLoS One. [Internet] 2016 [cited 2017 Jan 02];2;11(12)[10p].

8. Nowak M, Krolak-Nowak K, Sobolewska-Wlodarczyk A, Fichna J, Wlodarczyk M. Elevated risk of venous thromboembolic events in patients with inflammatory myopathies. Vasc Health Risk Manag 2016 Jun 3;12:233-8.

9. Kubota Y, McAdams-DeMarco M, Folsom AR. Serum uric acid, gout, and venous thromboembolism: The atherosclerosis risk in communities study. Thromb Res 2016 Aug;144:144-8. DOI: 10.1016/j.thromres.2016.06.020

10. Silvestri E, Scalera A, Emmi G, Squatrito D, Ciucciarelli L, Cenci C, et al. Thrombosis in Autoimmune Diseases: A Role for Immunosuppressive Treatments? Semin Thromb Hemost 2016 Sep;42(6):650-61. DOI: 10.1055/s-0036-1579642

11. Ungprasert P, Wijarnpreecha K, Tanratana P. Risk of venous thromboembolism in patients with celiac disease: A systematic review and meta-analysis. J Gastroenterol Hepatol 2016 Jul;31(7):1240-5. DOI: 10.1111/ jgh.13282

12. Lentz SR. Thrombosis in the setting of obesity or inflammatory bowel disease. Blood 2016 Nov;128(20):238894. DOI: 10.1182/blood-2016-05-716720

13. Horvei LD, Grimnes G, Hindberg K, Mathiesen EB, Njolstad I, Wilsgaard T, et al. C-reactive protein, obesity, and the risk of arterial and venous thrombosis. J Thromb Haemost 2016 Aug;14(8):1561-71. DOI: 10.1111/jth. 13369

14. Christensen DH, Horvath-Puho E, Thomsen RW, Knudsen ST, Dekkers O, Prandoni P, et al. Venous thromboembolism and risk of cancer in patients with diabetes mellitus. J Diabetes Complications 2016 MayJun;30(4):603-7. DOI: 10.1016/j.jdiacomp.2016.01.011 
15. Matos MF, Lourenco DM, Orikaza CM, Bajerl JAH, Noguti MAE, Morelli VM. The role of IL-6, IL-8 and MCP-1 and their promoter polymorphisms IL-6 $-174 \mathrm{GC}$, IL-8 -251AT and MCP-1 -2518AG in the risk of venous thromboembolism: A case-control study. Thromb Res 2011;128:216-20. DOI: 10.1016/j.thromres.2011.04.016

16. Pabinger I, Thaler J, Ay C. Biomarkers for prediction of venous thromboembolism in cancer. Blood 2013;122(12):2011-8. DOI: 10.1182/ blood-2013-04-460147

17. Reitsma PH, Rosendaal FR. Activation of innate immunity in patients with venous thrombosis: the Leiden Thrombophilia Study. J Thromb Haemost 2004;2:61922. DOI: $10.1111 / \mathrm{j} .1538-7836.2004 .00689 . \mathrm{x}$

18. Roumen-Klappe EM, den Heijer M, van Uum SHM, van der Ven-Jongekrijg J, van der Graaf F, Wollersheim $\mathrm{H}$. Inflammatory response in the acute phase of deep vein thrombosis. J Vasc Surg 2002;35:701-6. DOI: 10.1067/mva.2002.121746

19. Saghazadeh A, Hafizi S, Rezaei N. Inflammation in venous thromboembolism: Cause or consequence? Int Immunopharmacol 2015 Sep;28(1):655-65. DOI: 10.1016/j.intimp.2015.07.044

20. Saghazadeh A, Rezaei N. Inflammation as a cause of venous thromboembolism. Crit Rev Oncol Hematol 2016 Mar;99:272-85. DOI: 10.1016/j.critrevonc. 2016.01 .007

21. van Aken,BE, Reitsma PH, Rosendaal FR. Interleukin 8 and venous thrombosis: evidence for a role of inflammation in thrombosis. Br J Haematol 2002;116: 173-7. DOI: $10.1046 /$ j.1365-2141.2002.03245.x

22. Zagorski J, Gellar MA, Obraztsova M, Kline JA, Watts JA. Inhibition of CINC-1 Decreases Right Ventricular Damage Caused by Experimental Pulmonary Embolism in Rats. J Immunol 2007;179:7820-6. DOI: 10.4049/jimmunol.179.11.7820

23. Rabinovich A, Cohen JM, Cushman M, Kahn SR. Association between inflammation biomarkers, anatomic extent of deep venous thrombosis, and venous symptoms after deep venous thrombosis. J Vasc Surg Venous Lymphat Disord 2015 Oct;3(4):347-53. DOI: 10.1016/j.jvsv.2015.04.005

24. Jacobs B, Obi A, Wakefield T. Diagnostic biomarkers in venous thromboembolic disease. J Vasc Surg Venous Lymphat Disord 2016 Oct;4(4):508-17. DOI: 10.1016/j.jvsv.2016.02.005

25. Cushman M, O’Meara ES, Heckbert SR, Zakai NA, Rosamond W, Folsom AR. Body size measures, hemostatic and inflammatory markers and risk of venous thrombosis: The Longitudinal Investigation of Thromboembolism Etiology. Thromb Res 2016 Aug;144:12732. DOI: 10.1016/j.thromres.2016.06.012

26. Cushman M, Callas PW, Allison MA, Criqui MH.
Inflammation and peripheral venous disease. The San Diego Population Study. Thromb Haemost 2014;112(3):566-72. DOI: 10.1160/TH13-10-0860

27. Hou H, Ge Z, Ying P, Dai J, Shi D, Xu Z, et al. Biomarkers of deep venous thrombosis. J Thromb Thrombolysis 2012 Oct;34(3):335-46. DOI: 10.1007/s11239012-0721-y

28. Eagleton MJ, Henke PK, Luke CE, Hawley AE, Bedi A, Knipp BS, et al. Inflammation and intimal hyperplasia associated with experimental pulmonary embolism. J Vasc Surg 2002;36:581-8. DOI: 10.1067/ mva.2002.126556

29. Wakefield TW, Myers DD, Henke PK. Mechanisms of Venous Thrombosis and Resolution. Arterioscler Thromb Vasc Biol 2008;28:387-91. DOI: 10.1161/ATVBAHA.108.162289

30. Lang IM, Dorfmuller P, Vonk Noordegraaf A. The Pathobiology of Chronic Thromboembolic Pulmonary Hypertension. Ann Am Thorac Soc 2016 Jul;13 Suppl 3:S215-21. DOI: 10.1513/AnnalsATS.201509-620AS

31. Roumen-Klappe EM, Janssen MCH, van Rossum J, Holewijn S, van Bokhoven MMJA, Kaasjager K, et al. Inflammation in deep vein thrombosis and the development of post-thrombotic syndrome: a prospective study. J Thromb Haemost 2009;7:582-7. DOI: 10.1111/j.1538-7836.2009.03286.x

32. Pesavento R, Prandoni P. Prevention and treatment of the post-thrombotic syndrome and of the chronic thromboembolic pulmonary hypertension. Expert Rev Cardiovasc Ther 2015 Feb;13(2):193-207. DOI: 10.1586/14779072.2015.1000306

33. Evans CE, Grover SP, Humphries J, Saha P, Patel AP, Patel AS, et al. Antiangiogenic Therapy Inhibits Venous Thrombus Resolution. Arterioscler Thromb Vasc Biol 2014;34:565-570. DOI: 10.1161/ATVBAHA. 113.302998

34. Choi KJ, Cha SI, Shin KM, Lim JK, Yoo SS, Lee J, et al. Factors determining clot resolution in patients with acute pulmonary embolism. Blood Coagul Fibrinolysis 2016 Apr;27(3):294-300. DOI: 10.1097/ MBC.0000000000000425

35. Alias S, Redwan B, Panzenbock A, Winter MP, Schubert U, Voswinckel R, et al. Defective angiogenesis delays thrombus resolution: a potential pathogenetic mechanism underlying chronic thromboembolic pulmonary hypertension. Arterioscler Thromb Vasc Biol 2014 Apr;34(4):810-9. DOI: 10.1161/ATVBAHA.113.302991

36. Souza-Costa DC, Zerbini T, Palei AC, Gerlach RF, Tanus-Santos JE. L-arginine Attenuates Acute Pulmonary Embolism-Induced Increases in Lung Matrix Metalloproteinase-2 and Matrix Metalloproteinase-9. Chest 2005;128:3705-10. DOI: 10.1378/chest.128.5.3705 